\title{
Some more long continued fractions, I
}

\author{
by \\ James Mc Laughlin and Peter Zimmer (West Chester, PA)
}

1. Introduction. Let $D\left(x_{1}, \ldots, x_{r}, k\right)$ be a polynomial in $\mathbb{Z}\left[x_{1}, \ldots, x_{r}\right]$, where $x_{1}, \ldots, x_{r}$ are integer variables and $k$ is a positive integer parameter which appears as an exponent in the expression of $D\left(x_{1}, \ldots, x_{r}, k\right)$. Suppose further that there are positive lower bounds $T_{1}$ and $T_{2}$ such that, for all integral $x_{i} \geq T_{1}$ and all integral $k \geq T_{2}$, the surd $\sqrt{D\left(x_{1}, \ldots, x_{r}, k\right)}$ has a regular continued fraction of the form

$$
\sqrt{D\left(x_{1}, \ldots, x_{r}, k\right)}=\left[a_{0} ; \overline{a_{1}, \ldots, a_{n_{k}}, 2 a_{0}}\right],
$$

where each $a_{j}:=a_{j}\left(x_{1}, \ldots, x_{r}, k\right) \in \mathbb{Z}\left[x_{1}, \ldots, x_{r}\right]$ for $j=0,1, \ldots, n_{k}$, and the length of the period, $n_{k}+1$, depends only on $k$ ( $k$ may also be present in some of the $a_{j}$ as an exponent). Under these circumstances we say that $D\left(x_{1}, \ldots, x_{r}, k\right)$ has a long continued fraction expansion and call the expansion $\left[a_{0} ; \overline{a_{1}, \ldots, a_{n_{k}}, 2 a_{0}}\right]$ a long continued fraction.

We give the following example due to Madden [11] to illustrate the concept. Let $b, n$ and $k$ be positive integers. Set

$$
D:=D(b, n, k):=\left(b(1+2 b n)^{k}+n\right)^{2}+2(1+2 b n)^{k} .
$$

Then

$$
\begin{aligned}
& \sqrt{D}=\left[b(1+2 b n)^{k}+n ; \overline{b, 2 b(1+2 b n)^{k-1}, b(2 b n+1), 2 b(2 b n+1)^{k-2}},\right. \\
& \overline{b(2 b n+1)^{2}, 2 b(2 b n+1)^{k-3}, \ldots, b(1+2 b n)^{k-1}, 2 b,} \\
& \overline{n+b(2 b n+1)^{k}} \text {, } \\
& \overline{2 b, b(1+2 b n)^{k-1}, \ldots, 2 b(2 b n+1)^{k-3}, b(2 b n+1)^{2},} \\
& \left.\overline{2 b(2 b n+1)^{k-2}, b(2 b n+1), 2 b(1+2 b n)^{k-1}, b, 2\left(n+b(1+2 b n)^{k}\right)}\right] \text {. }
\end{aligned}
$$

2000 Mathematics Subject Classification: Primary 11A55; Secondary 11R27.

Key words and phrases: continued fractions, Pell's equation, quadratic fields.

The authors would like to thank the referee of an earlier version of this paper for pointing out a number of relevant papers, of which they were previously unaware. 
The fundamental period in the continued fraction expansion has length $4 k+2$.

Since the discovery of Daniel Shanks [19] and [20], there have been a number of examples of families of quadratic surds whose continued fraction expansions have unbounded period length. These include those discovered by Hendy [6], Bernstein [2] and [3], Williams [23], [22] and [21], Levesque and Rhin [8], Azuhatu [1], Levesque [7], Halter-Koch [4] and [5], Mollin and Williams [13] and [14], van der Poorten [17], and, more recently, Madden [11] and Mollin [12].

Williams' paper [23] contains several tables listing surds with long continued fraction expansions, along with the length of their fundamental period. In Mollin and Williams [13] and [14] and Williams [22] and [21], the authors describe a general method for computing the fundamental period $\pi(N)$ of the continued fraction expansion of $\sqrt{N}$, where

$$
N=\left(\sigma\left(q r a^{n}+\mu\left(a^{k}+\lambda\right) / q\right) / 2\right)^{2}-\sigma^{2} \mu \lambda a^{n} r .
$$

Here $\mu, \lambda \in\{-1,1\}, \sigma \in\{1,2\}$ (the value of $\sigma$ depends on the parity of $\left.q r a^{n}+\mu\left(a^{k}+\lambda\right) / q\right), q r \mid a^{k}+\lambda, \operatorname{gcd}(n, k)=1$, and $n>k \geq 1$. To describe this method we need some additional notation. For positive integers $r>1$ and $s$, consider the regular continued fraction expansion

$$
s / r=\left[q_{0} ; q_{1}, \ldots, q_{m}\right],
$$

with $q_{m}>1$. Define $M(r, s):=2\lfloor(m+1) / 2\rfloor$. For a fixed $Q$ and $a$ with $\operatorname{gcd}(a, Q)=1$, denote by $s_{i}$ the integer satisfying

$$
s_{i} \equiv a^{i}(\bmod Q),
$$

with $1 \leq s_{i}<Q$. Let $\omega=\omega(a, Q)$ denote the multiplicative order of $a$ modulo $Q$ and define

$$
W(a, q)=\sum_{i=1}^{\omega} M\left(s_{i}, Q\right) .
$$

The authors give various formulae for $\pi(N)$, formulae that depend on the various parameters and the functions $W(\cdot, \cdot)$ and $\omega(\cdot, \cdot)$.

In this present paper we use matrix methods (based on Madden's method in [11]) to derive several infinite families of quadratic surds with long continued fraction expansions. We feel that, while these matrix methods may not be as widely applicable, where they can be used the proofs are more transparent, more direct and less intricate than proofs by the method outlined above in the papers of Mollin and Williams. Furthermore, some of the families of long continued fractions in the present paper generalize some of the results in some of the papers cited previously in this introduction. 
2. Preliminaries. We first recall some basic properties of continued fractions. For any sequence of numbers $a_{0}, a_{1}, \ldots$, define, for $i \geq 0$, the numbers $A_{i}$ and $B_{i}$ (the $i$ th numerator convergent and $i$ th denominator convergent, respectively, of the continued fraction below) by

$$
a_{0}+\frac{1}{a_{1}}+\frac{1}{a_{2}}+\cdots+\frac{1}{a_{i}}=\frac{A_{i}}{B_{i}} .
$$

By the correspondence between matrices and continued fractions, we have

$$
\left(\begin{array}{cc}
a_{0} & 1 \\
1 & 0
\end{array}\right)\left(\begin{array}{cc}
a_{1} & 1 \\
1 & 0
\end{array}\right) \cdots\left(\begin{array}{cc}
a_{i} & 1 \\
1 & 0
\end{array}\right)=\left(\begin{array}{cc}
A_{i} & A_{i-1} \\
B_{i} & B_{i-1}
\end{array}\right) .
$$

It is also well known that

$$
A_{i}=a_{i} A_{i-1}+A_{i-2}, \quad B_{i}=a_{i} B_{i-1}+B_{i-2} .
$$

See [10], for example, for these basic properties of continued fractions.

Our starting point is the following elementary result.

Lemma 1. Let $q_{0}, q_{1}, \ldots, q_{1}, q_{0}$ be a finite palindromic sequence of positive integers (with or without a central term) and let

$$
\left(\begin{array}{cc}
q_{0} & 1 \\
1 & 0
\end{array}\right)\left(\begin{array}{cc}
q_{1} & 1 \\
1 & 0
\end{array}\right) \cdots\left(\begin{array}{cc}
q_{1} & 1 \\
1 & 0
\end{array}\right)\left(\begin{array}{cc}
q_{0} & 1 \\
1 & 0
\end{array}\right)=:\left(\begin{array}{ll}
w & u \\
u & v
\end{array}\right) .
$$

Then

$$
\sqrt{w / v}=\left[q_{0} ; \overline{q_{1}, q_{2}, \ldots, q_{2}, q_{1}, 2 q_{0}}\right] .
$$

Proof. As usual, $\overline{q_{1}, q_{2}, \ldots, q_{2}, q_{1}, 2 q_{0}}$ means that the sequence of partial quotients $q_{1}, q_{2}, \ldots, q_{2}, q_{1}, 2 q_{0}$ is repeated infinitely often. Note that the matrix on the right side of (2.3) is symmetric, since the left side is a symmetric product of symmetric matrices.

Let $\alpha=\left[q_{0} ; \overline{q_{1}, q_{2}, \ldots, q_{2}, q_{1}, 2 q_{0}}\right]$, so that $\alpha=\left[q_{0} ; q_{1}, q_{2}, \ldots, q_{2}, q_{1}, q_{0}+\alpha\right]$. Then, by (2.3), (2.1) and (2.2), we have

$$
\alpha=\frac{w+\alpha u}{u+\alpha v},
$$

so that $\alpha^{2}=w / v$ and the result follows.

We will be primarily interested in the case where $w / v$ is an integer. The next result, although entirely trivial, is also central to what follows.

Lemma 2. Let $D$ be any complex number and let $\alpha$ and $\beta$ be any complex numbers such that $\alpha+\beta \neq 0$. Define the matrix $P$ by

$$
P=\left(\begin{array}{cc}
-\sqrt{D} & \sqrt{D} \\
1 & 1
\end{array}\right)\left(\begin{array}{cc}
\alpha & 0 \\
0 & \beta
\end{array}\right)\left(\begin{array}{cc}
-\sqrt{D} & 1 \\
\sqrt{D} & 1
\end{array}\right) .
$$


Then

$$
\frac{P_{1,1}}{P_{2,2}}=D \text {. }
$$

To investigate the fundamental units in the corresponding real quadratic fields $\mathbb{Q}(\sqrt{D})$, there is the following theorem on page 119 of [15]:

THEOREM 1. Let $D$ be a square-free, positive rational integer and let $K=\mathbb{Q}(\sqrt{D})$. Denote by $\varepsilon_{0}$ the fundamental unit of $K$ which exceeds unity, by $s$ the period of the continued fraction expansion for $\sqrt{D}$, and by $P / Q$ the $(s-1)$ th approximant of it.

If $D \not \equiv 1(\bmod 4)$ or $D \equiv 1(\bmod 8)$, then

$$
\varepsilon_{0}=P+Q \sqrt{D}
$$

However, if $D \equiv 5(\bmod 8)$, then

$$
\varepsilon_{0}=P+Q \sqrt{D} \quad \text { or } \quad \varepsilon_{0}^{3}=P+Q \sqrt{D} .
$$

Finally, the norm of $\varepsilon_{0}$ is positive if the period $s$ is even and negative otherwise.

This theorem implies the following result.

Proposition 1. Let $D$ be a non-square positive integer, $D \not \equiv 5(\bmod 8)$. Suppose $\sqrt{D}=\left[q_{0} ; \overline{q_{1}, \ldots, q_{1}, 2 q_{0}}\right]$, and that

$$
\begin{aligned}
\left(\begin{array}{cc}
q_{0} & 1 \\
1 & 0
\end{array}\right)\left(\begin{array}{cc}
q_{1} & 1 \\
1 & 0
\end{array}\right) & \cdots\left(\begin{array}{cc}
q_{1} & 1 \\
1 & 0
\end{array}\right)\left(\begin{array}{cc}
q_{0} & 1 \\
1 & 0
\end{array}\right) \\
= & \left(\begin{array}{ll}
w & u \\
u & v
\end{array}\right)=\left(\begin{array}{cc}
-\sqrt{D} & \sqrt{D} \\
1 & 1
\end{array}\right)\left(\begin{array}{cc}
\alpha & 0 \\
0 & \beta
\end{array}\right)\left(\begin{array}{cc}
-\sqrt{D} & 1 \\
\sqrt{D} & 1
\end{array}\right) .
\end{aligned}
$$

Then the fundamental unit in $\mathbb{Q}(\sqrt{D})$ is $2 \sqrt{D} \beta$.

Proof. By Theorem 1 and (2.1), the fundamental unit in $\mathbb{Q}(\sqrt{D})$ is $u+$ $v \sqrt{D}$, and the equality of this quantity and $2 \sqrt{D} \beta$ follows from comparing corresponding entries in the matrices above.

REMARK. Other approaches can be used to calculate the fundamental unit in the case $D \equiv 5(\bmod 8)$, but we do not pursue that here.

We will follow Madden and let $\vec{N}$ denote the sequence $a_{1}, \ldots, a_{j}$ whenever

$$
N=\left(\begin{array}{cc}
a_{1} & 1 \\
1 & 0
\end{array}\right) \cdots\left(\begin{array}{cc}
a_{j} & 1 \\
1 & 0
\end{array}\right),
$$

and let $\overleftarrow{N}$ denote the sequence $a_{j}, a_{j-1}, \ldots, a_{2}, a_{1}$ 
3. Some general propositions. We next state several variants of a result of Madden from [11]. These general propositions will allow us to construct specific families of long continued fractions in the next section.

Proposition 2. Let $k, u, v, w$ and $r$ be positive integers such that $r w / v$ is an integer, and let $x$ be a rational such that $w x$ and $2 v x$ are integers. Let

$$
C=\left(\begin{array}{ll}
r & 0 \\
0 & 1
\end{array}\right) .
$$

Suppose further, for each integer $n \in\{0,1, \ldots, k-1\}$, that the matrix $N_{n}$ defined by

$$
N_{n}:=C^{-n}\left(\begin{array}{cc}
u & r^{k-1} v \\
w & r u-2 v w x
\end{array}\right) C^{n}=\left(\begin{array}{cc}
u & r^{k-1-n} v \\
r^{n} w & r u-2 v w x
\end{array}\right)
$$

has an expansion of the form

$$
N_{n}=\left(\begin{array}{cc}
a_{1}^{(n)} & 1 \\
1 & 0
\end{array}\right) \cdots\left(\begin{array}{cc}
a_{j_{n}}^{(n)} & 1 \\
1 & 0
\end{array}\right)
$$

where each $a_{i}^{(n)}$ is a positive integer. Then

$$
\begin{aligned}
& \sqrt{w r^{k} / v+w^{2} x^{2}} \\
& =\left[w x ; \overline{\vec{N}_{k-1}, \vec{N}_{k-2}, \ldots, \vec{N}_{1}, \vec{N}_{0}, 2 v x, \overleftarrow{N}_{0}, \overleftarrow{N}_{1}, \ldots, \overleftarrow{N}_{k-2}, \overleftarrow{N}_{k-1}, 2 w x}\right] .
\end{aligned}
$$

If $w r^{k} / v+w^{2} x^{2} \not \equiv 5(\bmod 8)$ and is square-free, then the fundamental unit in $\mathbb{Q}\left(w r^{k} / v+w^{2} x^{2}\right)$ is

$$
\frac{w\left(r u+v\left(-w x+\sqrt{w\left(r^{k}+v w x^{2}\right) / v}\right)\right)^{2 k}}{v\left(-w x+\sqrt{w\left(r^{k}+v w x^{2}\right) / v}\right)^{2}} .
$$

Proof. Let $D=w r^{k} / v+w^{2} x^{2}$. We consider the matrix product

$$
\left(\begin{array}{cc}
w x & 1 \\
1 & 0
\end{array}\right) N_{k-1} \cdots N_{1} N_{0}\left(\begin{array}{cc}
2 v x & 1 \\
1 & 0
\end{array}\right) N_{0}^{\mathrm{T}} N_{1}^{\mathrm{T}} \cdots N_{k-1}^{\mathrm{T}}\left(\begin{array}{cc}
w x & 1 \\
1 & 0
\end{array}\right) .
$$

By the definition of the $N_{n}$, this product equals

$$
\left(\begin{array}{cc}
w x & 1 \\
1 & 0
\end{array}\right) C^{-k}\left(C N_{0}\right)^{k}\left(\begin{array}{cc}
2 v x & 1 \\
1 & 0
\end{array}\right)\left(\left(C N_{0}\right)^{k}\right)^{\mathrm{T}} C^{-k}\left(\begin{array}{cc}
w x & 1 \\
1 & 0
\end{array}\right) .
$$

Define the matrix $M$ by

$$
M:=\left(\begin{array}{cc}
v x-\frac{v \sqrt{D}}{w} & v x+\frac{v \sqrt{D}}{w} \\
1 & 1
\end{array}\right) .
$$


One can check that

$$
\begin{aligned}
& \left(\begin{array}{cc}
w x & 1 \\
1 & 0
\end{array}\right) C^{-k}=\left(\begin{array}{cc}
-\sqrt{D} & \sqrt{D} \\
1 & 1
\end{array}\right)\left(\begin{array}{cc}
\frac{1}{-w x-\sqrt{D}} & 0 \\
0 & \frac{1}{-w x+\sqrt{D}}
\end{array}\right) M^{-1} \\
& \left(C N_{0}\right)^{k}=M\left(\begin{array}{cc}
r u-v w x-v \sqrt{D} & 0 \\
0 & r u-v w x+v \sqrt{D}
\end{array}\right)^{k} M^{-1} \\
& M^{-1}\left(\begin{array}{cc}
2 v x & 1 \\
1 & 0
\end{array}\right)\left(M^{-1}\right)^{\mathrm{T}}=\left(\begin{array}{cc}
\frac{-w}{2 v \sqrt{D}} & 0 \\
0 & \frac{w}{2 v \sqrt{D}}
\end{array}\right) .
\end{aligned}
$$

Thus

$$
\begin{aligned}
\left(\begin{array}{cc}
w x & 1 \\
1 & 0
\end{array}\right) C^{-k} & \left(C N_{0}\right)^{k}\left(\begin{array}{cc}
2 v x & 1 \\
1 & 0
\end{array}\right)\left(\left(C N_{0}\right)^{k}\right)^{\mathrm{T}} C^{-k}\left(\begin{array}{cc}
w x & 1 \\
1 & 0
\end{array}\right) \\
= & \left(\begin{array}{cc}
-\sqrt{D} & \sqrt{D} \\
1 & 1
\end{array}\right)\left(\begin{array}{cc}
\frac{1}{-w x-\sqrt{D}} & 0 \\
0 & \frac{1}{-w x+\sqrt{D}}
\end{array}\right) \\
& \times\left(\begin{array}{cc}
r u-v w x-v \sqrt{D} & 0 \\
0 & r u-v w x+v \sqrt{D}
\end{array}\right)^{k}\left(\begin{array}{cc}
\frac{-w}{2 v \sqrt{D}} & 0 \\
0 & \frac{w}{2 v \sqrt{D}}
\end{array}\right) \\
& \times\left(\begin{array}{cc}
r u-v w x-v \sqrt{D} & r u-v w x+v \sqrt{D}
\end{array}\right) \\
& \times\left(\begin{array}{cc}
\frac{1}{-w x-\sqrt{D}} & 0 \\
0 & \frac{1}{-w x+\sqrt{D}}
\end{array}\right)\left(\begin{array}{cc}
-\sqrt{D} & 1 \\
\sqrt{D} & 1
\end{array}\right) .
\end{aligned}
$$

The result follows by Lemmas 1, 2 and Proposition 1.

The fundamental period of the continued fractions above contains a central partial quotient, namely $2 v x$. We next show how to construct long continued fractions which do not have a central partial quotient.

Proposition 3. Let $u, v, x$ and $r$ be positive integers. Let

$$
C=\left(\begin{array}{ll}
r & 0 \\
0 & 1
\end{array}\right)
$$

Suppose further, for each integer $k \geq 1$ and each integer $n \in\{0,1, \ldots, k-1\}$, that the matrix $N_{n}$ defined by

$$
N_{n}:=C^{-n}\left(\begin{array}{cc}
u & r^{k-1} v \\
r^{k} v & r u-2 v x
\end{array}\right) C^{n}=\left(\begin{array}{cc}
u & r^{k-1-n} v \\
r^{k+n} v & r u-2 v x
\end{array}\right)
$$


has an expansion of the form

$$
N_{n}=\left(\begin{array}{cc}
a_{1}^{(n)} & 1 \\
1 & 0
\end{array}\right) \cdots\left(\begin{array}{cc}
a_{j_{n}}^{(n)} & 1 \\
1 & 0
\end{array}\right)
$$

where each $a_{i}^{(n)}$ is a positive integer. Then, for each integer $k \geq 1$,

$$
\begin{aligned}
& \sqrt{r^{2 k}+x^{2}} \\
& \quad=\left[x ; \overline{\vec{N}_{k-1}, \vec{N}_{k-2}, \ldots, \vec{N}_{1}, \vec{N}_{0}, \overleftarrow{N}_{0}, \overleftarrow{N}_{1}, \ldots, \overleftarrow{N}_{k-2}, \overleftarrow{N}_{k-1}, 2 x}\right] .
\end{aligned}
$$

If $r^{2 k}+x^{2} \not \equiv 5(\bmod 8)$ and is square-free, then the fundamental unit in $\mathbb{Q}\left(\sqrt{r^{2 k}+x^{2}}\right)$ is

$$
\left(x+\sqrt{r^{2 k}+x^{2}}\right)\left(u+v\left(-x+\sqrt{r^{2 k}+x^{2}}\right) / r\right)^{2 k} .
$$

Proof. We proceed as in the proof of Proposition 2. Let $D=r^{2 k}+x^{2}$. As above, the definition of $N_{n}$ implies

$$
\begin{aligned}
\left(\begin{array}{ll}
x & 1 \\
1 & 0
\end{array}\right) N_{k-1} \cdots N_{1} & N_{0} N_{0}^{\mathrm{T}} N_{1}^{\mathrm{T}} \cdots N_{k-1}^{\mathrm{T}}\left(\begin{array}{ll}
x & 1 \\
1 & 0
\end{array}\right) \\
& =\left(\begin{array}{ll}
x & 1 \\
1 & 0
\end{array}\right) C^{-k}\left(C N_{0}\right)^{k}\left(\left(C N_{0}\right)^{k}\right)^{\mathrm{T}} C^{-k}\left(\begin{array}{ll}
x & 1 \\
1 & 0
\end{array}\right) .
\end{aligned}
$$

Define the matrix $M$ by

$$
M:=\left(\begin{array}{cc}
\frac{x-\sqrt{D}}{r^{k}} & \frac{x+\sqrt{D}}{r^{k}} \\
1 & 1
\end{array}\right) .
$$

One can check that

$$
\begin{aligned}
& \left(\begin{array}{ll}
x & 1 \\
1 & 0
\end{array}\right) C^{-k}=\left(\begin{array}{cc}
-\sqrt{D} & \sqrt{D} \\
1 & 1
\end{array}\right)\left(\begin{array}{cc}
\frac{x-\sqrt{D}}{r^{2 k}} & 0 \\
0 & \frac{x+\sqrt{D}}{r^{2 k}}
\end{array}\right) M^{-1} \\
& \left(C N_{0}\right)^{k}=M\left(\begin{array}{cc}
r u-v x-v \sqrt{D} & 0 \\
0 & r u-v x+v \sqrt{D}
\end{array}\right)^{k} M^{-1}, \\
& M^{-1}\left(M^{-1}\right)^{\mathrm{T}}=\left(\begin{array}{cc}
\frac{1}{2}+\frac{x}{2 \sqrt{D}} & 0 \\
0 & \frac{1}{2}-\frac{x}{2 \sqrt{D}}
\end{array}\right) .
\end{aligned}
$$


Thus

$$
\begin{aligned}
& \left(\begin{array}{ll}
x & 1 \\
1 & 0
\end{array}\right) C^{-k}\left(C N_{0}\right)^{k}\left(\left(C N_{0}\right)^{k}\right)^{\mathrm{T}} C^{-k}\left(\begin{array}{ll}
x & 1 \\
1 & 0
\end{array}\right) \\
& =\left(\begin{array}{cc}
-\sqrt{D} & \sqrt{D} \\
1 & 1
\end{array}\right)\left(\begin{array}{cc}
\frac{x-\sqrt{D}}{r^{2 k}} & 0 \\
0 & \frac{x+\sqrt{D}}{r^{2 k}}
\end{array}\right) \\
& \times\left(\begin{array}{cc}
r u-v x-v \sqrt{D} & 0 \\
0 & r u-v x+v \sqrt{D}
\end{array}\right)^{k}\left(\begin{array}{cc}
\frac{1}{2}+\frac{x}{2 \sqrt{D}} & 0 \\
0 & \frac{1}{2}-\frac{x}{2 \sqrt{D}}
\end{array}\right) \\
& \times\left(\begin{array}{cc}
r u-v x-v \sqrt{D} & 0 \\
0 & \quad r u-v x+v \sqrt{D}
\end{array}\right)^{k} \\
& \quad \times\left(\begin{array}{cc}
\frac{x-\sqrt{D}}{r^{2 k}} & 0 \\
0 & \frac{x+\sqrt{D}}{r^{2 k}}
\end{array}\right)\left(\begin{array}{cc}
-\sqrt{D} & 1 \\
\sqrt{D} & 1
\end{array}\right),
\end{aligned}
$$

and once again the result follows by Lemmas 1 and 2 and Proposition 1.

It is also possible to create long continued fractions with no central partial quotient, but with two extra central partial quotients that do not come from $\vec{N}_{0}$ and $\overleftarrow{N}_{0}$

Proposition 4. Let $u, v, w, q$ and $r$ be positive integers. Let

$$
C=\left(\begin{array}{ll}
r & 0 \\
0 & 1
\end{array}\right)
$$

Suppose further, for each integer $k \geq 1$ and each integer $n \in\{0,1, \ldots, k-1\}$, that the matrix $N_{n}$ defined by

$$
\begin{aligned}
N_{n} & :=C^{-n}\left(\begin{array}{cc}
u & r^{k-1} v \\
v\left(r^{k}+4 q w\right) & r u-2 q r^{k} v-2\left(1+4 q^{2}\right) v w
\end{array}\right) C^{n} \\
& =\left(\begin{array}{cc}
u & r^{k-1-n} v \\
r^{n} v\left(r^{k}+4 q w\right) & r u-2 q r^{k} v-2\left(1+4 q^{2}\right) v w
\end{array}\right)
\end{aligned}
$$

has an expansion of the form

$$
N_{n}=\left(\begin{array}{cc}
a_{1}^{(n)} & 1 \\
1 & 0
\end{array}\right) \cdots\left(\begin{array}{cc}
a_{j_{n}}^{(n)} & 1 \\
1 & 0
\end{array}\right)
$$


where each $a_{i}^{(n)}$ is a positive integer. Then, for each integer $k \geq 1$,

$$
\sqrt{r^{k}\left(r^{k}+4 q w\right)+\left(w+q\left(r^{k}+4 q w\right)\right)^{2}}
$$

$=\left[w+q\left(r^{k}+4 q w\right) ;\right.$

$\left.\vec{N}_{k-1}, \vec{N}_{k-2}, \ldots, \vec{N}_{1}, \vec{N}_{0}, 2 q, 2 q, \overleftarrow{N}_{0}, \overleftarrow{N}_{1}, \ldots, \overleftarrow{N}_{k-2}, \overleftarrow{N}_{k-1}, 2\left(w+q\left(r^{k}+4 q w\right)\right)\right]$.

For ease of notation, set $D=r^{k}\left(r^{k}+4 q w\right)+\left(w+q\left(r^{k}+4 q w\right)\right)^{2}$ and $\gamma=$ $q\left(r^{k}+4 q w\right)$. Then the fundamental unit in $\mathbb{Q}(\sqrt{D})$ is

$$
\frac{(u+v(\sqrt{D}-w-\gamma) / r)^{2 k}(\sqrt{D}-w+\gamma)(\sqrt{D}+w+\gamma)^{2}}{\gamma^{2}} .
$$

Proof. We proceed as in the proof of Propositions 2 and 3. Let

$$
D=r^{k}\left(r^{k}+4 q w\right)+\left(w+q\left(r^{k}+4 q w\right)\right)^{2} .
$$

As above, the definition of $N_{n}$ implies

$$
\begin{aligned}
&\left(\begin{array}{cc}
w+q\left(r^{k}+4 q w\right) & 1 \\
1 & 0
\end{array}\right) N_{k-1} \cdots N_{1} N_{0}\left(\begin{array}{cc}
2 q & 1 \\
1 & 0
\end{array}\right) \\
& \times\left(\begin{array}{cc}
2 q & 1 \\
1 & 0
\end{array}\right) N_{0}^{\mathrm{T}} N_{1}^{\mathrm{T}} \cdots N_{k-1}^{\mathrm{T}}\left(\begin{array}{cc}
w+q\left(r^{k}+4 q w\right) & 1 \\
1 & 0
\end{array}\right) \\
&=\left(\begin{array}{cc}
w+q\left(r^{k}+4 q w\right) & 1 \\
1 & 0
\end{array}\right) C^{-k}\left(C N_{0}\right)^{k}\left(\begin{array}{cc}
2 q & 1 \\
1 & 0
\end{array}\right) \\
& \times\left(\begin{array}{cc}
2 q & 1 \\
1 & 0
\end{array}\right)\left(\left(C N_{0}\right)^{k}\right)^{\mathrm{T}} C^{-k}\left(\begin{array}{cc}
w+q\left(r^{k}+4 q w\right) & 1 \\
1 & 0
\end{array}\right) .
\end{aligned}
$$

Recall that $\gamma=q\left(r^{k}+4 q w\right)$ and define the matrix $M$ by

$$
M:=\left(\begin{array}{cc}
\frac{q(v(w+\gamma)-\sqrt{D} v)}{v \gamma} & \frac{q((w+\gamma) v+\sqrt{D} v)}{v \gamma} \\
1 & 1
\end{array}\right) .
$$

One can check (preferably with a computer algebra system like Mathematica) that 


$$
\begin{aligned}
& \left(\begin{array}{cc}
w+q\left(r^{k}+4 q w\right) & 1 \\
1 & 0
\end{array}\right) C^{-k} \\
& =\left(\begin{array}{cc}
-\sqrt{D} & \sqrt{D} \\
1 & 1
\end{array}\right)\left(\begin{array}{cc}
\frac{q r^{-k}(w+\gamma-\sqrt{D})}{\gamma} & 0 \\
0 & \frac{q r^{-k}(w+\gamma+\sqrt{D})}{\gamma}
\end{array}\right) M^{-1}, \\
& \left(C N_{0}\right)^{k}=M\left(\begin{array}{cc}
r u-v(w+\gamma+\sqrt{D}) & 0 \\
0 & r u+v(-w-\gamma+\sqrt{D})
\end{array}\right)^{k} M^{-1}, \\
& M^{-1}\left(\begin{array}{cc}
2 q & 1 \\
1 & 0
\end{array}\right)\left(\begin{array}{cc}
2 q & 1 \\
1 & 0
\end{array}\right)\left(M^{-1}\right)^{\mathrm{T}}=\left(\begin{array}{cc}
\frac{1}{2}+\frac{w-\gamma}{2 \sqrt{D}} & 0 \\
0 & \frac{1}{2}-\frac{w-\gamma}{2 \sqrt{D}}
\end{array}\right) .
\end{aligned}
$$

Thus, as in Propositions 2 and 3, the result follows by Lemmas 1 and 2 and Proposition 1.

4. Long continued fractions. We next give specific values (in terms of other variables) for some of the variables in the propositions so as to produce explicit long continued fractions. The main problem is to do this in such a way that the matrices $N_{n}$ satisfy (3.1), (3.3) or (3.5).

From Proposition 2, we have

$$
N_{0}=\left(\begin{array}{cc}
u & r^{k-1} v \\
w & r u-2 v w x
\end{array}\right)
$$

so one obvious approach is to initially define $u, v, w, x$ and $r$ from a product of the form

$$
N_{0}=\left(\begin{array}{cc}
u & r^{k-1} v \\
w & r u-2 v w x
\end{array}\right)=\left(\begin{array}{cc}
a_{1}^{(0)} & 1 \\
1 & 0
\end{array}\right) \cdots\left(\begin{array}{cc}
a_{j_{0}}^{(0)} & 1 \\
1 & 0
\end{array}\right)
$$

and then specialize the $a_{i}^{(0)}$ so that each $N_{n}$ also has an expansion of a similar form. We proceed similarly with Propositions 3 and 4.

We consider one example in detail to illustrate the method. We consider Proposition 2 with

$$
N_{0}=\left(\begin{array}{cc}
u & r^{k-1} v \\
w & r u-2 v w x
\end{array}\right)=\left(\begin{array}{ll}
a & 1 \\
1 & 0
\end{array}\right)\left(\begin{array}{ll}
b & 1 \\
1 & 0
\end{array}\right)=\left(\begin{array}{cc}
1+a b & a \\
b & 1
\end{array}\right) .
$$

Upon comparing $(1,2)$ entries, it can be seen that $a$ must be a multiple of $r^{k-1}$, so replace $a$ by $r^{k-1} a$ and we then have

$$
\left(\begin{array}{cc}
u & r^{k-1} v \\
w & r u-2 v w x
\end{array}\right)=\left(\begin{array}{cc}
1+r^{k-1} a b & r^{k-1} a \\
b & 1
\end{array}\right) .
$$


Then $v=a, w=b, u=1+a b r^{k-1}$ and $x=\left(a b r^{k}+r-1\right) /(2 a b)$. The requirements that $w x, w r / v$ and $2 v x$ be integers force $b$ and $r$ to have the forms $b=2 m a, r=1+2 a m s$, respectively, for some integers $m$ and $s$. Thus we finally have

$$
\begin{aligned}
& u=1+2 a^{2} m(1+2 a m s)^{-1+k}, \quad v=a, \quad w=2 a m, \\
& x=\frac{s+a(1+2 a m s)^{k}}{2 a} .
\end{aligned}
$$

With these values, we have

$$
\begin{aligned}
\frac{w r^{k}}{v} & +w^{2} x^{2}=m\left(2(1+2 a m s)^{k}+m\left(s+a(1+2 a m s)^{k}\right)^{2}\right), \\
N_{n} & =\left(\begin{array}{cc}
u & r^{k-1-n} v \\
w r^{n} & r u-2 v w x
\end{array}\right) \\
& =\left(\begin{array}{cc}
1+2 a^{2} m(1+2 a m s)^{-1+k} & a(1+2 a m s)^{-1+k-n} \\
2 a m(1+2 a m s)^{n} & 1
\end{array}\right) \\
& =\left(\begin{array}{cc}
a(1+2 a m s)^{-1+k-n} & 1 \\
1 & 0
\end{array}\right)\left(\begin{array}{cc}
2 a m(1+2 a m s)^{n} & 1 \\
1 & 0
\end{array}\right) .
\end{aligned}
$$

Thus

$$
\vec{N}_{n}=a(1+2 a m s)^{-1+k-n}, 2 a m(1+2 a m s)^{n} .
$$

For a non-square integer $D$, let $l(D)$ denote the length of the fundamental period in the regular continued fraction expansion of $\sqrt{D}$. We have proved the following theorem.

Theorem 2. Let $a, m, s$ and $k$ be positive integers. Set

$$
D:=m\left(2(1+2 a m s)^{k}+m\left(s+a(1+2 a m s)^{k}\right)^{2}\right) .
$$

Then $l(D)=4 k+2$ and

$$
\begin{aligned}
\sqrt{D}=\left[m\left(s+a(1+2 a m s)^{k}\right) ;\right. & \frac{\overline{a, 2 a m(1+2 a m s)^{k-1},}}{\frac{a(2 a m s+1), 2 a m(2 a m s+1)^{k-2},}{a(2 a m s+1)^{2}, 2 a m(2 a m s+1)^{k-3},}} \\
& \frac{}{\frac{a(1+2 a m s)^{k-1}, 2 a m,}{s+a(2 a m s+1)^{k},}} \\
& \frac{2 a m, a(1+2 a m s)^{k-1},}{2 a}
\end{aligned}
$$




$$
\begin{aligned}
& \overline{2 a m(2 a m s+1)^{k-3}, a(2 a m s+1)^{2}}, \\
& \overline{2 a m(2 a m s+1)^{k-2}, a(2 a m s+1)}, \\
& \overline{2 a m(1+2 a m s)^{k-1}, a,} \\
& \left.\overline{2 m\left(s+a(1+2 a m s)^{k}\right)}\right] .
\end{aligned}
$$

This long continued fraction generalizes Madden's first example in Section 3 of [11], where Madden's continued fraction is the case $m=1$ of the continued fraction above (this continued fraction of Madden is also given in row 1 of Table 3 in Williams' paper [23]). The case $m=1, a=1$ gives Bernstein's Theorem 3 from [2].

TheOREM 3. Let $a>1, m, s$ and $k$ be positive integers. Set

$$
D:=m\left(2(2 a m s-1)^{k}+m\left(s-a(2 a m s-1)^{k}\right)^{2}\right) .
$$

Then $l(D)=6 k+2$ and

$$
\begin{aligned}
\sqrt{D}=\left[m\left(-s+a(2 a m s-1)^{k}\right) ;\right. & \overline{a-1,1,2 a m(2 a m s-1)^{k-1}-1,} \\
& \frac{a(2 a m s-1)-1,1,2 a m(2 a m s-1)^{k-2}-1,}{a(2 a m s-1)^{2}-1,1,2 a m(2 a m s-1)^{k-3}-1,} \\
& \frac{\cdots}{a(-1+2 a m s)^{k-1}-1,1,2 a m-1,} \\
& \frac{-s+a(2 a m s-1)^{k},}{2 a m-1,1, a(1+2 a m s)^{k-1}-1,} \\
& \frac{}{2 a m(2 a m s-1)^{k-3}-1,1, a(2 a m s-1)^{2}-1,} \\
& \frac{2 a m(2 a m s-1)^{k-2}-1,1, a(1+2 a m s)-1,}{2 a m(2 a m s-1)^{k-1}-1,1, a-1,} \\
& \frac{\left.2 m\left(-s+a(2 a m s-1)^{k}\right)\right] .}{2 a m s}
\end{aligned}
$$

Proof. In Proposition 2, let

$$
\begin{aligned}
& u=2 a^{2} m(2 a m s-1)^{k-1}-1, \quad v=a, \quad w=2 a m, \quad r=2 a m s-1, \\
& x=\frac{a(2 a m s-1)^{k}-s}{2 a} .
\end{aligned}
$$


With these values,

$$
\begin{aligned}
& \frac{w r^{k}}{v}+w^{2} x^{2}=m\left(2(2 a m s-1)^{k}+m\left(s-a(2 a m s-1)^{k}\right)^{2}\right), \\
N_{n}= & \left(\begin{array}{cc}
u & r^{k-1-n} v \\
w r^{n} & r u-2 v w x
\end{array}\right) \\
= & \left(\begin{array}{cc}
-1+2 a^{2} m(2 a m s-1)^{-1+k} & a(2 a m s-1)^{-1+k-n} \\
2 a m(2 a m s-1)^{n} & 1
\end{array}\right) \\
= & \left(\begin{array}{cc}
a(1+2 a m s)^{k-n-1}-1 & 1 \\
1 & 0
\end{array}\right)\left(\begin{array}{cc}
1 & 1 \\
1 & 0
\end{array}\right)\left(\begin{array}{cc}
2 a m(1+2 a m s)^{n}-1 & 1 \\
1 & 0
\end{array}\right) .
\end{aligned}
$$

This example generalizes Madden's second example (p. 130 of [11]) (set $m=1$ ) (Madden's second example can also be found in row 2 of Table 3 in [23]), Theorem 8.1 of Levesque and Rhin [8] (set $a=2, m=a$ and $s=1$ ) and Theorem 10.1 of [8] ( $s e t a=2, m=a / 2$ and $s=2$ ).

The example given by van der Poorten in [17], namely

$$
D=\left(q a^{k}-\frac{a+1}{4 q}\right)^{2}+a^{k} \quad \text { with } 4 q \mid a+1,
$$

follows upon setting $a=2 q, m=1 / 2$ and $s=(a+1) /(2 q)$.

We can also let $a, m$ and $s$ take negative values in Theorem 2. Any zeroand negative partial quotients in the continued fraction expansion can be removed by the following transformations:

$$
[m, n, 0, p, \alpha]=[m, n+p, \alpha], \quad[m,-n, \alpha]=[m-1,1, n-1,-\alpha] .
$$

Out of the eight possible sign combinations for $a, m$ and $s$, only four lead to distinct polynomials, those in Theorems 2 and 3 (Theorem 3 could also have been proved by replacing $s$ by $-s$ for the case $k$ is even, and replacing $m$ by $-m$ in the case $k$ is odd) and two others.

Our first application of this transformation is to Theorem 3.

Corollary 1. Let $m, s$ and $k$ be positive integers such that $m s>1$. Set

$$
D:=m\left(2(2 m s-1)^{k}+m\left(s-(2 m s-1)^{k}\right)^{2}\right) .
$$

Then $l(D)=6 k-2$ and

$$
\begin{aligned}
\sqrt{D}=\left[m\left(-s+(2 m s-1)^{k}\right)+1 ;\right. & \frac{\overline{2 m(2 m s-1)^{k-1}-1,}}{} \\
& \frac{(2 m s-1)-1,1,2 m(2 m s-1)^{k-2}-1,}{(2 m s-1)^{2}-1,1,2 m(2 m s-1)^{k-3}-1,} \\
& \frac{\cdots}{(-1+2 m s)^{k-1}-1,1,2 m-1,}
\end{aligned}
$$




$$
\begin{aligned}
& \frac{-s+(2 m s-1)^{k},}{2 m-1,1,(1+2 m s)^{k-1}-1,} \\
& \frac{\ldots,}{2 m(2 m s-1)^{k-3}-1,1,(2 m s-1)^{2}-1,} \\
& \left.\frac{2 m(2 m s-1)^{k-2}-1,1,(1+2 m s)-1,}{2 m(2 m s-1)^{k-1}-1,2 m\left(-s+(2 m s-1)^{k}\right)+2}\right] .
\end{aligned}
$$

Proof. This follows upon letting $a=1$ in Theorem 3 and using (4.1) to remove the zeros resulting from the " $a-1$ " terms.

This continued fraction generalizes that in Theorem 2 of Bernstein [2] (set $m=1$ and $s=a+1$ ) and also that in Theorem 6.1 of Levesque and Rhin [8] (set $m=2 a$ and $s=1)$.

THEOREM 4. Let $a>1, m, s$ and $k$ be positive integers. Set

$$
D:=m\left(m\left(s+a(2 a m s-1)^{k}\right)^{2}-2(2 a m s-1)^{k}\right) .
$$

Then $l(D)=6 k+4$ and

$$
\begin{aligned}
\sqrt{D}=[m(s+a(2 a m s & \left.-1)^{k}\right)-1 ; \overline{1, a-1,2 a m(2 a m s-1)^{k-1}-1,} \\
& \frac{1, a(2 a m s-1)-1,2 a m(2 a m s-1)^{k-2}-1,}{1, a(2 a m s-1)^{2}-1,2 a m(2 a m s-1)^{k-3}-1,} \\
& \frac{}{1, a(2 a m s-1)^{k-1}-1,2 a m-1,} \\
& \frac{1, s+a(2 a m s-1)^{k}-2,1,}{2 a m-1, a(2 a m s-1)^{k-1}-1,1,} \\
& \frac{}{2 a m(2 a m s-1)^{k-3}-1, a(2 a m s-1)^{2}-1,1,} \\
& \frac{2 a m(2 a m s-1)^{k-2}-1, a(2 a m s-1)-1,1,}{2 a m(2 a m s-1)^{k-1}-1, a-1,1,} \\
& \frac{\left.2 m\left(s+a(2 a m s-1)^{k}\right)-2\right] .}{2 m(2 a m s},
\end{aligned}
$$

Proof. We consider the cases where $k$ is odd and $k$ is even separately. We first consider $k$ odd.

Replace $a$ by $-a$ and $k$ by $2 k+1$ in Theorem 2. Then

$$
D:=m\left(m\left(s+a(2 a m s-1)^{2 k+1}\right)^{2}-2(2 a m s-1)^{2 k+1}\right)
$$


and

$$
\begin{aligned}
& \sqrt{D}=\left[m\left(s+a(2 a m s-1)^{2 k+1}\right) ; \overline{-a,-2 a m(2 a m s-1)^{2 k}}\right. \\
& \overline{a(2 a m s-1), 2 a m(2 a m s-1)^{2 k-1}} \text {, } \\
& -a(2 a m s-1)^{2},-2 a m(2 a m s-1)^{2 k-2}, \\
& \overline{a(2 a m s-1)^{2 k-1}, 2 a m(2 a m s-1)}, \\
& -a(2 a m s-1)^{2 k},-2 a m \text {, } \\
& \overline{s+a(2 a m s-1)^{2 k+1},} \\
& \overline{-2 a m,-a(2 a m s-1)^{2 k}} \\
& \overline{2 a m(2 a m s-1), a(2 a m s-1)^{2 k-1}} \text {, } \\
& \text {..., } \\
& \overline{-2 a m(2 a m s-1)^{2 k-2},-a(2 a m s-1)^{2}}, \\
& \overline{2 a m(2 a m s-1)^{2 k-1}, a(2 a m s-1)} \text {, } \\
& \overline{-2 a m(2 a m s-1)^{2 k},-a,} \\
& \left.\overline{2 m\left(s+a(2 a m s-1)^{2 k+1}\right)}\right] \text {. }
\end{aligned}
$$

We now apply the second identity at (4.1) repeatedly to get

$$
\begin{aligned}
\sqrt{D}=\left[m\left(s+a(2 a m s-1)^{2 k+1}\right)-1 ; \overline{1, a-1,2 a m(2 a m s-1)^{2 k}-1,}\right. & \frac{\cdots}{1, a(2 a m s-1)-1,2 a m(2 a m s-1)^{2 k-1}-1,} \\
& \frac{1, a(2 a m s-1)^{2}-1,2 a m(2 a m s-1)^{2 k-2}-1,}{\cdots} \\
& \frac{1, a(2 a m s-1)^{2 k}-1,2 a m-1,}{1, s+a(2 a m s-1)^{2 k+1}-2,1,} \\
& \frac{1,2 a m-1, a(2 a m s-1)^{2 k}-1,}{1,2 a m(2 a m s-1)^{2 k-2}-1, a(2 a m s-1)^{2}-1}, \\
& \frac{1,2 a m(2 a m s-1)^{2 k-1}-1, a(2 a m s-1)-1,}{1,2 a m(2 a m s-1)^{2 k}-1, a-1,} \\
& \frac{\left.2 m\left(s+a(2 a m s-1)^{2 k+1}\right)-2\right] .}{2 m}
\end{aligned}
$$

It is clear that $l(D)=12 k+10$. This proves the theorem for odd $k$. 
We next consider the case where $k$ is even. Replace $a$ by $-a, s$ by $-s$, $m$ by $-m$ and $k$ by $2 k$ in Theorem 2 . Then

$$
D:=m\left(m\left(s+a(2 a m s-1)^{2 k}\right)^{2}-2(2 a m s-1)^{2 k}\right)
$$

and

$$
\begin{aligned}
\sqrt{D}=\left[m\left(s+a(2 a m s-1)^{2 k}\right) ;\right. & \frac{-a,-2 a m(2 a m s-1)^{2 k-1},}{a(2 a m s-1), 2 a m(2 a m s-1)^{2 k-2},} \\
& \frac{\cdots}{-a(2 a m s-1)^{2},-2 a m(2 a m s-1)^{2 k-3},} \\
& \frac{}{a(2 a m s-1)^{2 k-2}, 2 a m(2 a m s-1),} \\
& \frac{}{-a(2 a m s-1)^{2 k-1},-2 a m,} \\
& \frac{-2 a m,-a(2 a m s-1)^{2 k-1},}{2 a m(2 a m s-1), a(2 a m s-1)^{2 k-2},} \\
& \frac{}{2 a m}, \\
& \frac{2 a m(2 a m s-1)^{2 k-3},-a(2 a m s-1)^{2},}{2 a m(2 a m s-1)^{2 k-2}, a(2 a m s-1),} \\
& \frac{2 a m(2 a m s-1)^{2 k-1},-a,}{\left.2 m\left(s+a(2 a m s-1)^{2 k}\right)\right] .}
\end{aligned}
$$

We again apply the second identity at (4.1) repeatedly and the stated continued fraction expansion follows. It is clear that $l(D)=12 k+4$ in this case. This completes the proof for even $k$.

This theorem generalizes Theorem 7.1 of Levesque and Rhin [8] (set $a=2, s=1$ and $m=a$ ) and Theorem 9.1 of Levesque and Rhin [8] (set $a=2, s=2$ and $m=a / 2$ ). Williams' example in row 3 of Table 3 in [23] is the case $m=1$ of the continued fraction above.

REMARK. It seems likely from the common form of the expansions that there should be an alternative proof of Theorem 4 that covers the even and odd cases simultaneously. However, we do not pursue that here.

Corollary 2. Let $m, s$ and $k$ be positive integers. Set

$$
D:=m\left(m\left(s+(2 m s-1)^{k}\right)^{2}-2(2 m s-1)^{k}\right) .
$$


Then $l(D)=6 k$ and

$$
\begin{aligned}
\sqrt{D}=[m(s+(2 m s- & \frac{\left.1)^{k}\right)-1 ; \overline{2 m(2 m s-1)^{k-1}},}{1,(2 m s-1)-1,2 m(2 m s-1)^{k-2}-1,} \\
& \frac{\cdots}{1,(2 m s-1)^{2}-1,2 m(2 m s-1)^{k-3}-1,} \\
& \frac{}{1,(2 m s-1)^{k-1}-1,2 m-1,} \\
& \frac{1, s+(2 m s-1)^{k}-2,1,}{2 m-1,(2 m s-1)^{k-1}-1,1,} \\
& \frac{\cdots,}{2 m(2 m s-1)^{k-3}-1,(2 m s-1)^{2}-1,1,} \\
& \left.\frac{2 m(2 m s-1)^{k-2}-1,(2 m s-1)-1,1,}{2 m(2 m s-1)^{k-1}, 2 m\left(s+(2 m s-1)^{k}\right)-2}\right] .
\end{aligned}
$$

Proof. Let $a=1$ in Theorem 4 and use (4.1) to remove the zeros resulting from the " $a-1$ " terms.

This result generalizes that in Theorem 1 of Bernstein [2] (set $s=a+1$ and $m=1$ ) and also that in Theorem 5.1 of Levesque and Rhin [8] (set $s=1$ and $m=2 a$ ). It also generalizes the example in row 5 of Table 3 in [23].

Theorem 5. Let $a>2, m, s$ and $k$ be positive integers. Set

$$
D:=m\left(m\left(-s+a(1+2 a m s)^{k}\right)^{2}-2(1+2 a m s)^{k}\right) .
$$

Then $l(D)=8 k+4$ and

$$
\begin{aligned}
\sqrt{D}=\left[m\left(-s+a(1+2 a m s)^{k}\right)-1 ; \overline{1, a-2,1,2 a m(1+2 a m s)^{k-1}-2,}\right. \\
\frac{}{\frac{1, a(2 a m s+1)-2,1,2 a m(2 a m s+1)^{k-2}-2,}{1, a(2 a m s+1)^{2}-2,1,2 a m(2 a m s+1)^{k-3}-2,}} \\
\\
\frac{\cdots}{\frac{1, a(1+2 a m s)^{k-1}-2,1,2 a m-2,}{1,-s+a(2 a m s+1)^{k}-2,1,}} \\
\frac{\frac{2 a m-2,1, a(1+2 a m s)^{k-1}-2,1,}{\cdots,}}{2 a m(2 a m s+1)^{k-3}-2,1, a(2 a m s+1)^{2}-2,1,}
\end{aligned}
$$




$$
\begin{aligned}
& \overline{2 a m(2 a m s+1)^{k-2}-2,1, a(2 a m s+1)-2,1} \\
& \overline{2 a m(1+2 a m s)^{k-1}-2,1, a-2,1} \\
& \left.\overline{2 m\left(-s+a(1+2 a m s)^{k}\right)-2}\right]
\end{aligned}
$$

Proof. Replace $a$ by $-a$ and $m$ by $-m$ in Theorem 2 . Then

$$
D:=m\left(m\left(-s+a(2 a m s+1)^{k}\right)^{2}-2(2 a m s+1)^{k}\right)
$$

and

$$
\begin{aligned}
& \sqrt{D}=\left[m\left(-s+a(2 a m s+1)^{k}\right) ; \overline{-a, 2 a m(2 a m s+1)^{k-1}},\right. \\
& \overline{-a(2 a m s+1), 2 a m(2 a m s+1)^{k-2}} \\
& -a(2 a m s+1)^{2}, 2 a m(2 a m s+1)^{k-3}, \\
& \overline{-a(2 a m s-1)^{k-2}, 2 a m(2 a m s+1)}, \\
& -a(2 a m s+1)^{k-1}, 2 a m \text {, } \\
& \overline{s-a(2 a m s+1)^{k}} \text {, } \\
& \overline{2 a m,-a(2 a m s+1)^{k-1}} \\
& \overline{2 a m(2 a m s+1),-a(2 a m s+1)^{k-2},} \\
& \overline{2 a m(2 a m s+1)^{k-3},-a(2 a m s+1)^{2}}, \\
& \overline{2 a m(2 a m s+1)^{k-2},-a(2 a m s+1)} \text {, } \\
& \overline{2 a m(2 a m s-1)^{k-1},-a,} \\
& \left.\overline{2 m\left(-s+a(2 a m s+1)^{k}\right)}\right] \text {. }
\end{aligned}
$$

We again apply the second identity at (4.1) repeatedly and the stated continued fraction expansion follows. It is clear that $l(D)=8 k+4$ in this case. This completes the proof.

Williams' continued fraction in row 9 of Table 3 in [23] is the case $m=1$ of the theorem above.

Corollary 3. Let $m, s$ and $k>1$ be positive integers such that $m s>1$. Set

$$
D:=m\left(m\left(-s+(1+2 m s)^{k}\right)^{2}-2(1+2 m s)^{k}\right) .
$$

Then $l(D)=8 k$ and

$$
\sqrt{D}=\left[m\left(-s+(1+2 m s)^{k}\right)-2 ; \frac{\overline{1,2 m(1+2 m s)^{k-1}-3,}}{1,(2 m s+1)-2,1,2 m(2 m s+1)^{k-2}-2,}\right.
$$




$$
\begin{aligned}
& \frac{\begin{array}{l}
1,(2 m s+1)^{2}-2,1,2 m(2 m s+1)^{k-3}-2, \\
\cdots
\end{array}}{1,(1+2 m s)^{k-1}-2,1,2 m-2,} \\
& \frac{1,-s+(2 m s+1)^{k}-2,1,}{2 m-2,1,(1+2 m s)^{k-1}-2,1,} \\
& \frac{\cdots,}{2 m(2 m s+1)^{k-3}-2,1,(2 m s+1)^{2}-2,1}, \\
& \frac{2 m(2 m s+1)^{k-2}-2,1,(2 m s+1)-2,1,}{2 m(1+2 m s)^{k-1}-3,1,} \\
& \frac{2 m\left(-s+(1+2 m s)^{k}\right)-4}{2 m} .
\end{aligned}
$$

Proof. This follows after letting $a=1$ in Theorem 5 and using (4.1) to remove the resulting zeroes and negatives.

Williams' continued fraction in row 8 of Table 3 in [23] is the case $m=1$ of the corollary above.

Corollary 4. Let $k>2$ and $s$ be positive integers. Set

$$
D:=\left((1+2 s)^{k}-s\right)^{2}-2(1+2 s)^{k} .
$$

Then $l(D)=8 k-4$ and

$$
\begin{aligned}
& \sqrt{D}=\left[(1+2 s)^{k}-s-2 ; \overline{1,2(1+2 s)^{k-1}-3},\right. \\
& \overline{1,(2 s+1)-2,1,2(2 s+1)^{k-2}-2,} \\
& \overline{1,(2 s+1)^{2}-2,1,2(2 s+1)^{k-3}-2,} \\
& \overline{1,(1+2 s)^{k-2}-2,1,2(2 s+1)-2} \\
& \overline{1,(1+2 s)^{k-1}-2,2,} \\
& \overline{-s+(2 s+1)^{k}-2,} \\
& \overline{2,(1+2 s)^{k-1}-2,1} \text {, } \\
& \overline{2(2 s+1)-2,1,(1+2 s)^{k-1}-2,1}, \\
& \overline{2(2 s+1)^{k-3}-2,1,(2 s+1)^{2}-2,1}, \\
& \overline{2(2 s+1)^{k-2}-2,1,(2 s+1)-2,1}, \\
& \overline{2(1+2 s)^{k-1}-3,1,} \\
& \left.\overline{2\left(s+(1+2 s)^{k}\right)-4}\right] \text {. }
\end{aligned}
$$


Proof. This follows after letting $m=1$ in Corollary 3 and using (4.1) to remove the zero resulting from the " $2 m-2$ " terms.

The result above is Theorem 4 in Bernstein [2].

Corollary 5. Let $m, s$ and $k>1$ be positive integers. Set

$$
D:=m\left(m\left(-s+2(1+4 m s)^{k}\right)^{2}-2(1+4 m s)^{k}\right) .
$$

Then $l(D)=8 k$ and

$$
\begin{aligned}
& \sqrt{D}=\left[m\left(-s+2(1+4 m s)^{k}\right)-1 ; \overline{2,4 m(1+4 m s)^{k-1}-2},\right. \\
& 1,2(4 m s+1)-2,1,4 m(4 m s+1)^{k-2}-2, \\
& \overline{1,2(4 m s+1)^{2}-2,1,4 m(4 m s+1)^{k-3}-2,} \\
& \overline{1,2(1+4 m s)^{k-1}-2,1,4 m-2,} \\
& \overline{1,-s+2(4 m s+1)^{k}-2,1,} \\
& \overline{4 m-2,1,2(1+4 m s)^{k-1}-2,1} \text {, } \\
& \text {..., } \\
& \overline{4 m(4 m s+1)^{k-3}-2,1,2(4 m s+1)^{2}-2,1}, \\
& \overline{4 m(4 m s+1)^{k-2}-2,1,2(4 m s+1)-2,1}, \\
& \overline{4 m(1+4 m s)^{k-1}-2,2} \\
& \left.\overline{2 m\left(-s+2(1+4 m s)^{k}\right)-2}\right] \text {. }
\end{aligned}
$$

Proof. This follows after letting $a=2$ in Theorem 5 and using (4.1) to remove the zero resulting from the " $a-2$ " terms.

We next use Propositions 3 and 4 to construct families of long continued fractions with no central partial quotient in the fundamental period.

Theorem 6. Let $b, s$ and $k$ be positive integers. Set

$$
D:=(4 b s+1)^{k}+\left(b(4 b s+1)^{k}+s\right)^{2} .
$$

Then $l(D)=2 k+1$ and

$$
\begin{aligned}
& \sqrt{D}=\left[b(4 b s+1)^{k}+s ; \overline{2 b, 2 b(4 b s+1)^{k-1}},\right. \\
& \overline{2 b(4 b s+1), 2 b(4 b s+1)^{k-2},} \\
& \overline{2 b(4 b s+1)^{2}, 2 b(4 b s+1)^{k-3}} \text {, } \\
& \overline{2 b(4 b s+1)\lfloor(k-1) / 2\rfloor, 2 b(4 b s+1)^{k-1-\lfloor(k-1) / 2\rfloor},} \\
& \overline{2 b(4 b s+1)^{k-3}, 2 b(4 b s+1)^{2}},
\end{aligned}
$$




$$
\overline{\left.\frac{2 b(4 b s+1)^{k-2}, 2 b(4 b s+1)}{2 b(4 b s+1)^{k-1}, 2 b, 2\left(b(4 b s+1)^{k}+s\right)}\right]} .
$$

Here we understand the mid-point of the period to be situated just after the $2 b(4 b s+1)^{\lfloor(k-1) / 2\rfloor}$ term for $k$ odd, and just after the $2 b(4 b s+1)^{k-1-\lfloor(k-1) / 2\rfloor}$ term for $k$ even.

Proof. We first prove this for even $k$. In Proposition 3 set $v=2 b, r=$ $1+4 b s, u=1+4 b^{2}(1+4 b s)^{2 k-1}$ and $x=s+b(1+4 b s)^{2 k}$. Then

$$
D=(4 b s+1)^{2 k}+\left(b(4 b s+1)^{2 k}+s\right)^{2} .
$$

With these values also,

$$
\begin{aligned}
N_{n} & =\left(\begin{array}{cc}
u & r^{k-1-n} v \\
r^{k+n} v & r u-2 v x
\end{array}\right) \\
& =\left(\begin{array}{cc}
2 b(1+4 b s)^{k-1-n} & 1 \\
1 & 0
\end{array}\right)\left(\begin{array}{cc}
2 b(1+4 b s)^{k+n} & 1 \\
1 & 0
\end{array}\right),
\end{aligned}
$$

and so $\vec{N}_{n}=2 b(1+4 b s)^{k-1-n}, 2 b(1+4 b s)^{k+n}$. Thus

$$
\begin{aligned}
& \sqrt{D}=\left[b(4 b s+1)^{2 k}+s ; \overline{2 b, 2 b(4 b s+1)^{2 k-1}},\right. \\
& \overline{2 b(4 b s+1), 2 b(4 b s+1)^{2 k-2},} \\
& \overline{2 b(4 b s+1)^{2}, 2 b(4 b s+1)^{2 k-3},} \\
& \overline{2 b(4 b s+1)^{k-1}, 2 b(4 b s+1)^{k}}, \\
& \overline{2 b(4 b s+1)^{k}, 2 b(4 b s+1)^{k-1}} \text {, } \\
& \overline{2 b(4 b s+1)^{2 k-3}, 2 b(4 b s+1)^{2},} \\
& \overline{2 b(4 b s+1)^{2 k-2}, 2 b(4 b s+1)}, \\
& \left.\overline{2 b(4 b s+1)^{2 k-1}, 2 b, 2\left(b(4 b s+1)^{2 k}+s\right)}\right] \text {, }
\end{aligned}
$$

and the result follows for even $k$.

For odd $k$ we use Proposition 4 above, where we set $v=2 b, r=1+4 b s$, $u=1+4 b^{2}(1+4 b s)^{2 k}, q=b(1+4 b s)^{k}$ and $w=s$. Then

$$
D=(4 b s+1)^{2 k+1}+\left(b(4 b s+1)^{2 k+1}+s\right)^{2} .
$$

With these values also,

$$
\begin{aligned}
N_{n} & =\left(\begin{array}{cc}
u & r^{k-1-n} v \\
r^{n} v\left(r^{k}+4 q w\right) & r u-2 q r^{k} v-2\left(1+4 q^{2}\right) v w
\end{array}\right) \\
& =\left(\begin{array}{cc}
2 b(1+4 b s)^{k-1-n} & 1 \\
1 & 0
\end{array}\right)\left(\begin{array}{cc}
2 b(1+4 b s)^{k+n+1} & 1 \\
1 & 0
\end{array}\right),
\end{aligned}
$$


and so $\vec{N}_{n}=2 b(1+4 b s)^{k-1-n}, 2 b(1+4 b s)^{k+n+1}$. Thus

$$
\begin{aligned}
\sqrt{D}=\left[b(4 b s+1)^{2 k+1}+s ;\right. & \frac{2 b, 2 b(4 b s+1)^{2 k},}{2 b(4 b s+1), 2 b(4 b s+1)^{2 k-1},} \\
& \frac{\cdots}{2 b(4 b s+1)^{2}, 2 b(4 b s+1)^{2 k-2},} \\
& \frac{\ldots}{2 b(4 b s+1)^{k-1}, 2 b(4 b s+1)^{k+1}}, \\
& \frac{2 b(4 b s+1)^{k}, 2 b(4 b s+1)^{k},}{2 b(4 b s+1)^{k+1}, 2 b(4 b s+1)^{k-1},} \\
& \frac{\cdots,}{2 b(4 b s+1)^{2 k-2}, 2 b(4 b s+1)^{2},} \\
& \left.\frac{2 b(4 b s+1)^{2 k-1}, 2 b(4 b s+1),}{2 b(4 b s+1)^{2 k}, 2 b, 2\left(b(4 b s+1)^{2 k+1}+s\right)}\right],
\end{aligned}
$$

and the result again follows.

The result above (without the explicit continued fraction expansion) appears in row 1 of Table 2 in [23].

As previously, we can let $b$ or $r$ take negative integral values and produce a new long continued fraction.

Corollary 6. Let $b, s$ and $k$ be positive integers. Set

$$
D:=(4 b s-1)^{2 k}+\left(b(4 b s-1)^{2 k}-s\right)^{2} .
$$

Then $l(D)=6 k+1$ and

$$
\begin{aligned}
\sqrt{D}=\left[b(4 b s-1)^{2 k}-s ;\right. & \frac{2 b-1,1,2 b(4 b s-1)^{2 k-1}-1,}{2 b(4 b s-1)-1,1,2 b(4 b s-1)^{2 k-2}-1,} \\
& \frac{\cdots}{2 b(4 b s-1)^{2}-1,1,2 b(4 b s-1)^{2 k-3}-1,} \\
& \frac{2 b(4 b s-1)^{k-1}-1,1,2 b(4 b s-1)^{k}-1,}{2 b(4 b s-1)^{k}-1,1,2 b(4 b s-1)^{k-1}-1,} \\
& \frac{\cdots,}{2 b(4 b s-1)^{2 k-3}-1,2 b(4 b s-1)^{2}-1,} \\
& \left.\frac{2 b(4 b s-1)^{2 k-2}-1,1,2 b(4 b s-1)-1,}{2 b(4 b s-1)^{2 k-1}-1,1,2 b-1,2\left(b(4 b s-1)^{2 k}-s\right)}\right] .
\end{aligned}
$$

Proof. This follows from Theorem 6 in the case $k$ is even, after replacing $s$ by $-s$ and using (4.1) to remove the resulting negative partial quotients from the resulting continued fraction expansion. 
Corollary 7. Let $b, s$ and $k$ be positive integers. Set

$$
D:=\left(b(4 b s-1)^{2 k+1}+s\right)^{2}-(4 b s-1)^{2 k+1} .
$$

Then $l(D)=6 k+5$ and

$$
\begin{aligned}
& \sqrt{D}=\left[b(4 b s-1)^{2 k+1}+s-1 ; \overline{1,2 b-1,2 b(4 b s-1)^{2 k}-1},\right. \\
& \overline{1,2 b(4 b s-1)-1,2 b(4 b s-1)^{2 k-1}-1,} \\
& \overline{1,2 b(4 b s-1)^{2}-1,2 b(4 b s-1)^{2 k-2}-1}, \\
& \overline{1,2 b(4 b s-1)^{k-1}-1,2 b(4 b s-1)^{k+1}-1,} \\
& \overline{1,2 b(4 b s-1)^{k}-1,2 b(4 b s-1)^{k}-1,1}, \\
& \overline{2 b(4 b s-1)^{k+1}-1,2 b(4 b s-1)^{k-1}-1,1,} \\
& \overline{2 b(4 b s-1)^{2 k-2}-1,2 b(4 b s-1)^{2}-1,1}, \\
& \overline{2 b(4 b s-1)^{2 k-1}-1,2 b(4 b s-1)-1,1}, \\
& \left.\overline{2 b(4 b s-1)^{2 k}-1,2 b-1,1,2\left(b(4 b s-1)^{2 k+1}+s-2\right)}\right] .
\end{aligned}
$$

Proof. This follows from Theorem 6 in the case $k$ is odd, after replacing $b$ by $-b$ and using (4.1) to remove the resulting negative partial quotients from the resulting continued fraction expansion.

REMARK. Statements similar to those in Corollaries 6 and 7 hold if $2 k$ is replaced by $2 k+1$, but these results cannot be derived from Theorem 6 . All of these statements, without explicit continued fraction expansions, appear in Table 2 of [23].

5. Fundamental units in real quadratic fields. It is straightforward in many cases to compute the fundamental units in the real quadratic fields corresponding to the surds in the various theorems and corollaries. In what follows, we assume $D$ is square-free and $D \not \equiv 5(\bmod 8)$.

In Theorem 2, for example, where

$$
D=m\left(2(1+2 a m s)^{k}+m\left(s+a(1+2 a m s)^{k}\right)^{2}\right),
$$

it follows directly from Proposition 2 that the fundamental unit in $\mathbb{Q}(\sqrt{D})$ is

$$
\frac{2 m\left(1+a\left(\sqrt{D}+m\left(s+a(1+2 a m s)^{k}\right)\right)\right)^{2 k}}{\left(\sqrt{D}-m\left(s+a(1+2 a m s)^{k}\right)\right)^{2}} .
$$

With $m=3, a=5, s=7$ and $k=2$, for example, we get $D=446005190022$ and the fundamental unit in $\mathbb{Q}(\sqrt{446005190022})$ is (after simplifying)

149199899813252915906267542273 
Likewise, in Theorem 6 (for even $k$ ), where

$$
D=(1+4 b s)^{2 k}+\left(s+b(1+4 b s)^{2 k}\right)^{2},
$$

Proposition 3 gives that the fundamental unit in $\mathbb{Q}(\sqrt{D})$ is

$$
\begin{gathered}
\frac{1}{(1+4 b s)^{2 k}}\left(s+b(1+4 b s)^{2 k}+\sqrt{(1+4 b s)^{2 k}+\left(s+b(1+4 b s)^{2 k}\right)^{2}}\right) \\
\times\left(-2 b\left(s+b(1+4 b s)^{2 k}\right)+(1+4 b s)\left(1+4 b^{2}(1+4 b s)^{-1+2 k}\right)\right. \\
\left.+2 b \sqrt{(1+4 b s)^{2 k}+\left(s+b(1+4 b s)^{2 k}\right)^{2}}\right)^{2 k} .
\end{gathered}
$$

Setting $s=3, b=5$, and $k=2$, for example, gives $D=4792683254153105$ and that the fundamental unit in $\mathbb{Q}(\sqrt{4792683254153105})$ is

18375851029288260766491636025698114848

$$
+265434944781468068474213871001 \sqrt{4792683254153105} .
$$

For our last example, we also consider Theorem 6 (for odd $k$ ), where

$$
D=(1+4 b s)^{2 k+1}+\left(s+b(1+4 b s)^{2 k+1}\right)^{2},
$$

Proposition 4 implies that the fundamental unit in $\mathbb{Q}(\sqrt{D})$ is

$$
\begin{aligned}
\frac{1}{(1+4 b s)^{2 k}} & \left(1+2 b \sqrt{D}+2 b s+2 b^{2}(1+4 b s)^{1+2 k}\right)^{2 k} \\
& \times\left(\sqrt{D}+s+4 b^{3}(1+4 b s)^{1+4 k}+b(1+4 b s)^{2 k}(3+4 b(\sqrt{D}+2 s))\right) .
\end{aligned}
$$

Letting $s=5, b=2$, and $k=1$ gives $D=19001864330$ and shows that the fundamental unit in $\mathbb{Q}(\sqrt{19001864330})$ is

$$
2682318982172034563+19458632525153 \sqrt{19001864330} .
$$

6. Concluding remarks. While considering the various long continued fractions in the papers by Bernstein [2], [3], Levesque and Rhin [8] and Williams [23], and trying to see if any more of the continued fractions in these papers could be generalized by the methods in the present paper, we were led to a new construction.

This new construction succeeds where the methods in the present paper fail, in that it allowed us to generalize some more of the continued fractions in the papers mentioned above.

We will investigate this new construction in a subsequent paper.

\section{References}

[1] T. Azuhatu, On the fundamental units and class numbers of real quadratic fields, II, Tokyo J. Math. 10 (1987), 259-270. 
[2] L. Bernstein, Fundamental units and cycles in the period of real quadratic number fields. I, Pacific J. Math. 63 (1976), 37-61.

[3] - Fundamental units and cycles in the period of real quadratic number fields. II, ibid., 63-78.

[4] F. Halter-Koch, Einige periodische Kettenbruchentwicklungen und Grundeinheiten quadratischer Ordnungen, Abh. Math. Sem. Univ. Hamburg 59 (1989), 157-169.

[5] —, Reell-quadratische Zahlkörper mit großer Grundeinheit, ibid., 171-181.

[6] M. D. Hendy, Applications of a continued fraction algorithm to some class number problems, Math. Comp. 28 (1974), 267-277.

[7] C. Levesque, Continued fraction expansions and fundamental units, J. Math. Phys. Sci. 22 (1988), 11-44.

[8] C. Levesque and G. Rhin, A few classes of periodic continued fractions, Util. Math. 30 (1986), 79-107.

[9] R. Lidl and H. Niederreiter, Finite Fields, Addison-Wesley, Reading, MA, 1983.

[10] L. Lorentzen and H. Waadeland, Continued Fractions with Applications, Stud. Comput. Math. 3, North-Holland, Amsterdam, 1992.

[11] D. J. Madden, Constructing families of long continued fractions, Pacific J. Math. 198 (2001), 123-147.

[12] R. A. Mollin, Construction of families of long continued fractions revisited, Acta Math. Acad. Paedagog. Nyházi. (N.S.) 19 (2003), 175-181.

[13] R. A. Mollin and H. C. Williams, On the period length of some special continued fractions, Sém. Théor. Nombres Bordeaux (2) 4 (1992), 19-42.

[14] -, -, Consecutive powers in continued fractions, Acta Arith. 61 (1992), 233-264.

[15] W. Narkiewicz, Elementary and Analytic Theory of Algebraic Numbers, 2nd ed., PWN and Springer, Warszawa and Berlin, 1990.

[16] O. Perron, Über Summengleichungen und Poincarésche Differenzengleichungen, Math. Ann. 84 (1921), 1-15.

[17] A. J. van der Poorten, Explicit formulas for units in certain quadratic number fields, in: Algorithmic Number Theory (Ithaca, NY, 1994), Lecture Notes in Comput. Sci. 877, Springer, Berlin, 1994, 194-208.

[18] A. J. van der Poorten and H. C. Williams, On certain continued fraction expansions of fixed period length, Acta Arith. 89 (1999), 23-35.

[19] D. Shanks, On Gauss's class number problems, Math. Comp. 23 (1969), 151-163.

[20] —, Class number, a theory of factorization, and genera, in: Proc. Sympos. Pure Math. 20, Amer. Math. Soc., Providence, RI, 1971, 415-440.

[21] H. C. Williams, A number theoretic function arising from continued fractions, Fibonacci Quart. 38 (2000), 201-211.

[22] - Some generalizations of the $S_{n}$ sequence of Shanks, Acta Arith. 69 (1995), 199215.

[23] - A note on the period length of the continued fraction expansion of certain $\sqrt{D}$, Util. Math. 28 (1985), 201-209.

Mathematics Department

Anderson Hall

West Chester University

West Chester, PA 19383, U.S.A.

E-mail: jmclaughl@wcupa.edu

pzimmer@wcupa.edu 